\title{
Opening the way to molecular cycloaddition of large molecules on supported silicene
}

Régis Stephan, Marie-Christine Hanf, and Philippe Sonnet

Citation: The Journal of Chemical Physics 143, 154706 (2015); doi: 10.1063/1.4933369

View online: https://doi.org/10.1063/1.4933369

View Table of Contents: http://aip.scitation.org/toc/jcp/143/15

Published by the American Institute of Physics

\section{Articles you may be interested in}

The study of electronic structure and properties of silicene for gas sensor application AIP Conference Proceedings 1719, 030039 (2016); 10.1063/1.4943734

First-principles design of silicene/ $\mathrm{Sc}_{2} \mathrm{CF}_{2}$ heterojunction as a promising candidate for field effect transistor Journal of Applied Physics 117, 085306 (2015); 10.1063/1.4913480

Origin of Dirac-cone-like features in silicon structures on $\mathrm{Ag}(111)$ and $\mathrm{Ag}(110)$

Journal of Applied Physics 114, 113710 (2013); 10.1063/1.4821339

Infrared absorbance of silicene and germanene

Applied Physics Letters 100, 261906 (2012); 10.1063/1.4731626

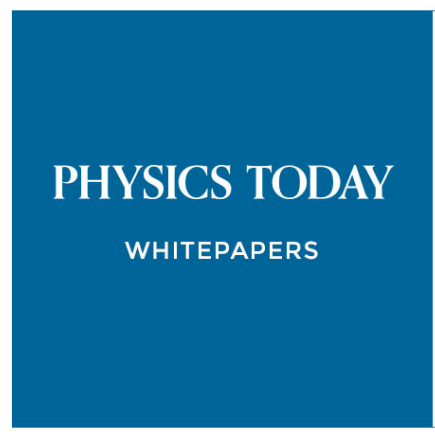




\title{
Opening the way to molecular cycloaddition of large molecules on supported silicene
}

\author{
Régis Stephan, Marie-Christine Hanf, and Philippe Sonnet ${ }^{a)}$ \\ Institut de Science des Matériaux de Mulhouse IS2M UMR 7361 CNRS-Université de Haute Alsace, \\ 3 bis rue Alfred Werner, 68057 Mulhouse, France
}

(Received 1 June 2015; accepted 5 October 2015; published online 21 October 2015)

\begin{abstract}
Within density functional theory, the adsorption of the $\mathrm{H}_{2} \mathrm{Pc}$ molecule on the $(3 \times 3)$ silicene $/(4 \times 4)$ $\mathrm{Ag}(111)$ surface has been investigated. We observe an electronic redistribution in the central macrocycle of the $\mathrm{H}_{2} \mathrm{Pc}$ molecule and the formation of two $\mathrm{Si}-\mathrm{N}$ covalent bonds between the molecule and the silicene, in agreement with a cycloaddition reaction. However, while on $\mathrm{SiC}(0001)(3 \times 3)$ or $\mathrm{Si}(111)(\sqrt{3} \times \sqrt{3}) R 30^{\circ}$-boron, the $\mathrm{H}_{2} \mathrm{Pc}$ molecule remains planar, and the $\mathrm{H}_{2} \mathrm{Pc}$ molecule takes a butterfly conformation on the silicene/Ag substrate due to an electrostatic or a polarization repulsion between the molecule and the silicene. Our study opens a way to the experimental adsorption of large organic molecules on supported silicene. (C) 2015 AIP Publishing LLC. [http://dx.doi.org/10.1063/1.4933369]
\end{abstract}

\section{INTRODUCTION}

Diels and Alder described for the first time in 1928 a specific reaction between a conjugated diene and a substituted alkene to form a substituted cyclohexene system. This reaction, named Diels-Alder reaction, is particularly useful in synthetic organic chemistry in solution. ${ }^{1-4}$

At the beginning of the 1990s, seminal papers have been dedicated to the study of elementary surface chemical processes on semiconductor surfaces, in particular, the adsorption of unsaturated hydrocarbons $\left(\mathrm{C}_{2} \mathrm{H}_{2}, \mathrm{C}_{2} \mathrm{H}_{4}, \ldots\right)$. Experimental results showed that the reaction occurs by a di- $\sigma$-bonding mechanism involving the formation of two $\mathrm{Si}-\mathrm{C}$ bonds..$^{5-14}$

In 1995, Liu and Hoffmann studied the chemisorption of acetylene on the $\mathrm{Si}(001)$ surface within different theoretical methods. ${ }^{15}$ The authors suggested the formation of a disilacyclobutene structure by a cycloaddition reaction, via a $\pi$ complex precursor and a biradical intermediate. The cycloaddition will be the surface reaction analog of the Diels-Alder reaction of organic chemistry. ${ }^{16,17}$

Then, the group of Hamers studied intensively, by means of Scanning Tunneling Microscopy (STM), Fourier Transform Infrared (FTIR) spectroscopy, and X-ray Photoelectron Spectroscopy (XPS), the interaction of small unsaturated organic molecules with the $\mathrm{Si}(001)$ surface. They emphasized the possibility of a [2+2] or a [4+2] cycloaddition reaction with the formation of two di- $\sigma$ bonds between the molecule and the surface. $^{15,18-25}$

More recently, in the context of the functionalization of wide bandgap semiconductor surfaces, the large metal-free phthalocyanine molecule has been anchored on the $\mathrm{SiC}(0001)$ $(3 \times 3)$ surface. Within STM and density functional theory (DFT) studies, the authors have proposed a reaction mechanism assimilated to a $[10+2]$ pseudo-cycloaddition via the formation of two $\mathrm{Si}-\mathrm{N}$ bonds involving the molecular conju-

\footnotetext{
a)Electronic mail: philippe.sonnet@uha.fr
}

gated macrocycle system and two neighbouring Si adatoms of the SiC surface. ${ }^{26,27}$ Another large molecule, namely, the PTCDI (perylene-3, 4:9, 10-bis (dicarboximide)) molecule, can be chemisorbed on $\mathrm{SiC}(0001)(3 \times 3)$. This molecule forms two $\mathrm{Si}-\mathrm{O}$ bonds between the $\mathrm{O}$ atoms of the molecule and two neighbouring $\mathrm{Si}$ adatoms of the substrate. This reaction is assimilated to a [12+2] pseudo-cycloaddition. ${ }^{28,29}$

In 2014, a new assisted molecular cycloaddition has been proposed within a predictive DFT-D2 study for the $\mathrm{H}_{2} \mathrm{Pc}$ molecule on $\operatorname{Si}(111)(\sqrt{3} \times \sqrt{3}) R 30^{\circ}$-boron in presence of one boron defect. ${ }^{30}$ In all papers, the calculations have shown that the $\mathrm{H}_{2} \mathrm{Pc}$ molecule remains planar after chemisorption. ${ }^{26,27,30}$

The cycloaddition reaction seems to be a general mechanism of molecular adsorption on surfaces presenting top silicon atoms, as the $\mathrm{Si}(001)(2 \times 1), \mathrm{Si}(111)(7 \times 7), \mathrm{Si}(111)$ $(\sqrt{3} \times \sqrt{3}) R 30^{\circ}$-boron, and Si-terminated $\operatorname{SiC}(0001)(3 \times 3)$ surfaces. The question now is whether the cycloaddition reaction could take place for large molecules on a silicene layer. Silicene is a structure with a hexagonal lattice which forms when a single layer of silicon atoms is deposited, for example, on a Ag substrate. ${ }^{31-44}$ The discovery of this new material in 2010 has led silicene to be considered as a promising novel material for nanoelectronics. ${ }^{31}$ Silicene should naturally benefit from current Si-based research and development technology. Indeed, silicene is a new material with huge potential application toward further miniaturization for the electronic industry. For example, Tao et al. reported, recently, the fabrication of a field effect transistor (FET) from silicene that can operate at room temperature. ${ }^{45}$ The silicene, formed by only one Si layer, could present the ultimate thickness for a transistor. Moreover, due to the specific electronic behavior of freestanding silicene, the study of the adsorption of small gas molecules $\left(\mathrm{NO}_{2}, \mathrm{O}_{2}, \mathrm{SO}_{2}\right.$, $\mathrm{NO}$, and $\mathrm{NH}_{3}$ ) on this system demonstrates potential applications as a gas sensor or for the development of metal-freecatalysis. ${ }^{46}$ Silicene bandgap engineering is the key element of these studies. The authors show that a bandgap for freestanding silicene is opened under $\mathrm{NO}$ and $\mathrm{NH}_{3}$ adsorption. ${ }^{46}$ 
However, silicene has been experimentally obtained only deposited on a substrate, in particular on $\operatorname{Ag}(111)$. Now, the silicene/Ag system presents electronic properties that are different from those of the usual $\operatorname{Si}(001)(2 \times 1)$ surface. Thus, the reactivity of the silicene/Ag system with respect to a large organic molecule is still unknown, and the possible functionalization of the silicene/Ag system remains a topic to explore. Finally, due to its specific electronic structure, it seems difficult to predict the behavior of the silicene/Ag system without any calculation or experiment.

In this paper, we have modelled, by means of DFT calculations, the possible chemisorption of a large molecule $\left(\mathrm{H}_{2} \mathrm{Pc}\right)$ on the $(3 \times 3)$ silicene $/(4 \times 4) \operatorname{Ag}(111)$ surface via a cycloaddition reaction. To our knowledge, no adsorption experiment or predictive calculation for such a large molecule on silicene has yet been published.

\section{THEORETICAL METHODS}

To perform periodic DFT calculations, the Vienna $A b$ initio Simulation Package (VASP) has been employed. ${ }^{47-50}$ The projector augmented wave (PAW) ${ }^{51,52}$ and generalized gradient approximation (GGA) potentials, with the PerdewBurke-Ernzerhof (PBE) exchange-correlation functional, have been used to carry out total energy calculations and perform geometry optimizations. ${ }^{53,54}$

In order to improve the description of the long-range interactions, the DFT-D2 method of Grimme as implemented in VASP has been employed ${ }^{55-57}$ This approximation has been already successfully used in the case of the $\mathrm{H}_{2} \mathrm{Pc}$ adsorption on the $\operatorname{SiC}(0001)(3 \times 3)$ and the $\operatorname{Si}(111)(\sqrt{3} \times \sqrt{3}) R 30^{\circ}$-boron surfaces (noted $\mathrm{SiC}$ and $\mathrm{SiB}$, respectively). ${ }^{26,27,30}$ In order to compare the adsorption of $\mathrm{H}_{2} \mathrm{Pc}$ on silicene/Ag with that on the surfaces of $\mathrm{SiC}$, passivated $\mathrm{SiB}$, and $\mathrm{SiB}$ with two defects (a boron defect is the substitution of one boron atom by a silicon one), the same parameters have been used in the present study. In particular, the integration of the Brillouin zone was reduced to the $\Gamma \mathrm{k}$-point. The cut-off energy for plane-wave is equal to $400 \mathrm{eV}$ corresponding to the carbon atom one.

The slab of the silicene/Ag substrate is modeled by one layer of silicon deposited on four layers of $\mathrm{Ag}$. When the $\mathrm{H}_{2} \mathrm{Pc}$ molecule is adsorbed on the silicene/Ag system, the total number of atoms is equal to 386 . The slab size is $(23.53 \AA$ $\times 23.53 \AA \times 43 \AA$ ). Due to the size of the silicene/Ag slab, we cannot study the molecular coverage effect. The number of layers in the present slab is similar to that used in a previous work. ${ }^{30}$ The $\mathrm{H}_{2} \mathrm{Pc}$ molecule and the four top-most surface layers were allowed to relax during structural optimization, which is obtained when the residual forces are equal to $0.02 \mathrm{eV} / \AA$. The rest of the atoms in the slab were fixed at their bulk-optimized lattice positions.

The charge transfer occurring in the $\mathrm{H}_{2} \mathrm{Pc} /$ silicene/Ag system is evaluated through the Bader scheme. ${ }^{58,59}$ The precision of the Bader charge using the $(240 \times 240 \times 420)$ grid is estimated to be better than $4 \times 10^{-4}$ electrons.

The electron localization function (noted ELF) is a dimensionless function in 3D space, where the value 1 corresponds to localized electrons and 0.5 to delocalized electrons. The ELF function has been often used to characterize the bonded interactions for a variety of materials ranging from insulators to metals. ${ }^{38,60-64}$

The adsorption energy $\left(E_{a d s}\right)$ is calculated as the difference between the total energy of the optimized $\mathrm{H}_{2} \mathrm{Pc} /$ silicene/Ag system $\left(E_{\mathrm{H}_{2} \mathrm{Pc} / \text { silicene/Ag }}\right)$ and the sum of the relaxed clean silicene/Ag substrate $\left(E_{\text {silicene } / A g}\right)$ and the $\mathrm{H}_{2} \mathrm{Pc}$ molecule in gas phase $\left(E_{\mathrm{H}_{2} P c}\right)$ energies, following this equation,

$$
E_{\text {ads }}=E_{\mathrm{H}_{2} P c / \text { silicene } / \mathrm{Ag}}-\left(E_{\text {silicene } / \mathrm{Ag}}+E_{\mathrm{H}_{2} P c}\right) \text {. }
$$

The molecule-substrate interaction energy is obtained by calculating the difference between the energy of the fully relaxed system $\left(\mathrm{H}_{2} \mathrm{Pc} / \mathrm{Si} / \mathrm{Ag}\right)$ and the isolated fragments $\left(\mathrm{H}_{2} \mathrm{Pc}\right.$ and $\mathrm{Si} / \mathrm{Ag}$ ) without further relaxation by the following formula:

$E_{\text {int }}=E_{\mathrm{H}_{2} P c / \text { silicene } / \mathrm{Ag}}-\left(E_{\text {fixed silicene } / \mathrm{Ag}}+E_{\text {fixed } \mathrm{H}_{2} \mathrm{P}_{c}}\right)$,

where $E_{\text {fixed } \mathrm{H}_{2} P_{c}}$ and $E_{\text {fixed silicene } / A g}$ correspond, respectively, to the energy of the optimized $\mathrm{H}_{2} \mathrm{Pc}$ molecule and $\mathrm{Si} / \mathrm{Ag}$ substrate fixed in the fully relaxed $\mathrm{H}_{2} \mathrm{Pc} /$ silicene/Ag system. We emphasize that the interaction energy does not contain the deformation energy. Part of the data has been treated with the visual molecular dynamics software developed by the Theoretical and Computational Biophysics Group in the Beckman Institute for Advanced Science and Technology at the University of Illinois at Urbana-Champaign. ${ }^{65,66}$ The VESTA software has been used to display the electron localization function map. ${ }^{67}$

\section{RESULTS AND DISCUSSION}

\section{A. Structural study}

The pristine silicene surface corresponds to the $(3 \times 3)$ silicene $/(4 \times 4) \operatorname{Ag}(111)$ phase, which is the most studied one and also the most stable. ${ }^{34,37,39}$ For this silicene layer, six Si (denoted top $\mathrm{Si}$ atom and indicated by striped yellow balls in Fig. 1) in eighteen are lifted with respect to the other $\mathrm{Si}$ (bottom $\mathrm{Si}$ atoms). Different distances between two top $\mathrm{Si}$ atoms of the silicene can be measured, ranging between $3.87 \AA$ and $11.25 \AA$. As already observed, the cycloaddition reaction is favourable for various reasons, and in particular, if the molecule $\mathrm{N} \cdots \mathrm{N}$ distance is close enough to the $\mathrm{Si} \cdot \mathrm{Si}$ distance of the substrate, in order to allow the formation of two $\mathrm{Si}-\mathrm{N}$ bonds. Moreover, previous studies on $\mathrm{SiC}$ and $\mathrm{SiB}$ with two boron defects have shown that the nitrogen atoms bond to $\mathrm{Si}$ adatoms, that is, lying above the surface. ${ }^{26,27,30}$ Now, the distance between two nitrogen atoms of the $\mathrm{H}_{2} \mathrm{Pc}$ molecule is $6.78 \AA$. In agreement with this distance, there are only three possible adsorption sites for the $\mathrm{H}_{2} \mathrm{Pc}$ molecule on silicene layer. Figures 1(a)-1(c) correspond to sites numbers 1, 2, and 3, with top $\mathrm{Si}-\mathrm{Si}$ distances (before molecule adsorption) of $9.13 \AA, 8.14 \AA$, and $7.90 \AA$, respectively. For the other sites involving top $\mathrm{Si}$ atoms, the $\mathrm{Si}-\mathrm{Si}$ distances are too small or too large with respect to the $\mathrm{N}-\mathrm{N}$ one to form $\mathrm{Si}-\mathrm{N}$ covalent bonds. This is also the case for the sites located at down $\mathrm{Si}$ atoms, as they would result in unrealistically large $\mathrm{Si}-\mathrm{N}$ distances. Moreover, we have tested the possibility to rotate the molecule by $90^{\circ}$ for all models. In Figure 1(d), we present, as an example, the rotation of the molecule in the site 2 . 


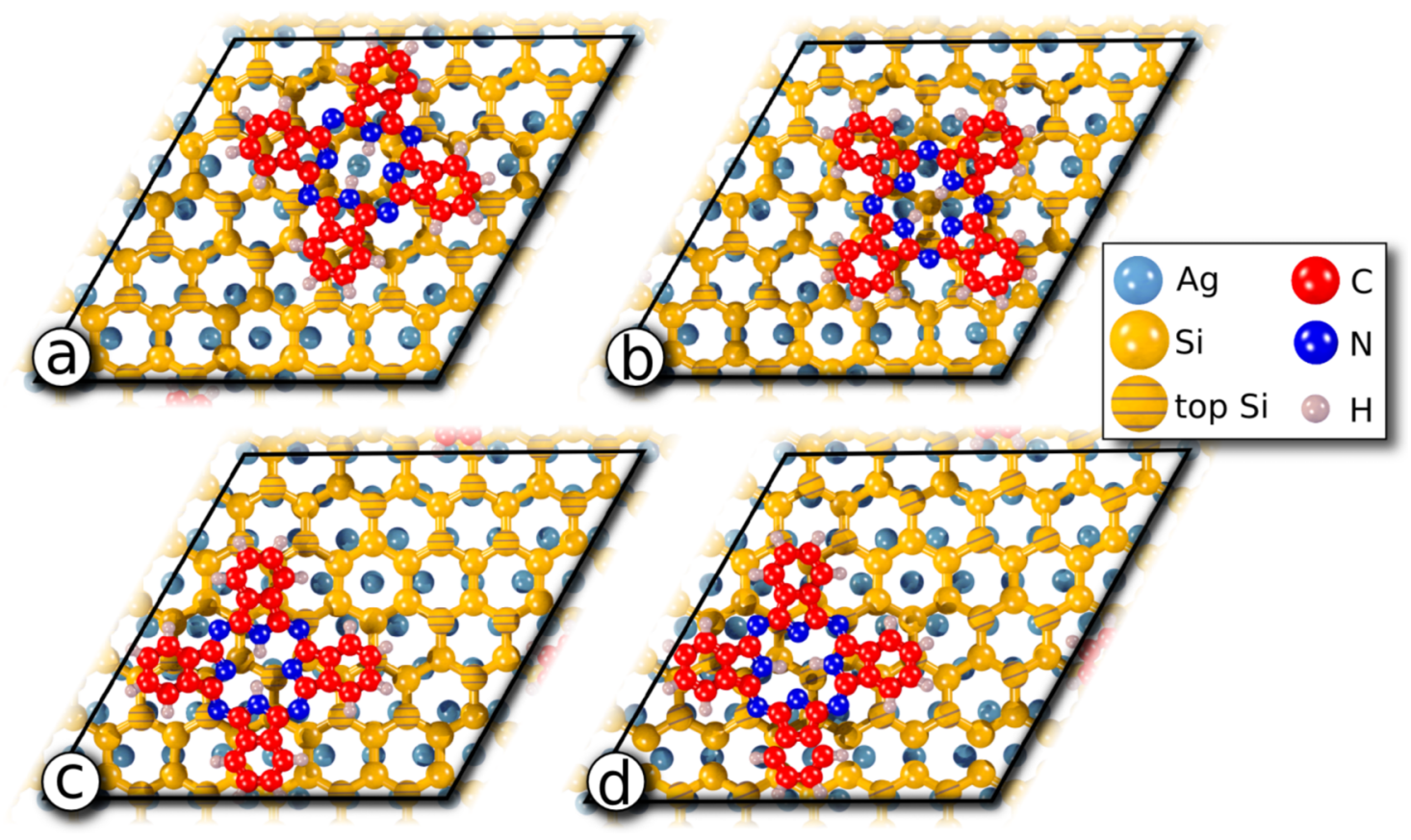

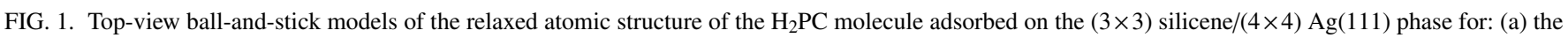
site 1 , (b) the site 3 , and (c) and (d) the site 2 in the two different orientations.

Figs. 2(a) and 2(b) present, for site 2, side-views of the adsorbed $\mathrm{H}_{2} \mathrm{Pc}$ molecule after relaxation. The results show that the $\mathrm{H}_{2} \mathrm{Pc}$ molecule is strongly deformed for all three sites and present characteristic angles $\alpha, \beta_{1}$, and $\beta_{2}$, whose values are displayed in Table I.

Now, let us take a look at the different atomic distances within the molecule and the substrate, displayed in Table I. N1 and $\mathrm{N} 2$ refer to the $\mathrm{N}$ atoms indicated in Fig. 2(b). Si1 and Si2 are the top Si atoms close to the N1 and N2 atoms, respectively. We find that, after relaxation, the $\mathrm{N} 1 \cdots \mathrm{N} 2$ distance increases slightly for the three sites, while the $\mathrm{Si} 1 \cdots \mathrm{Si} 2$ distance
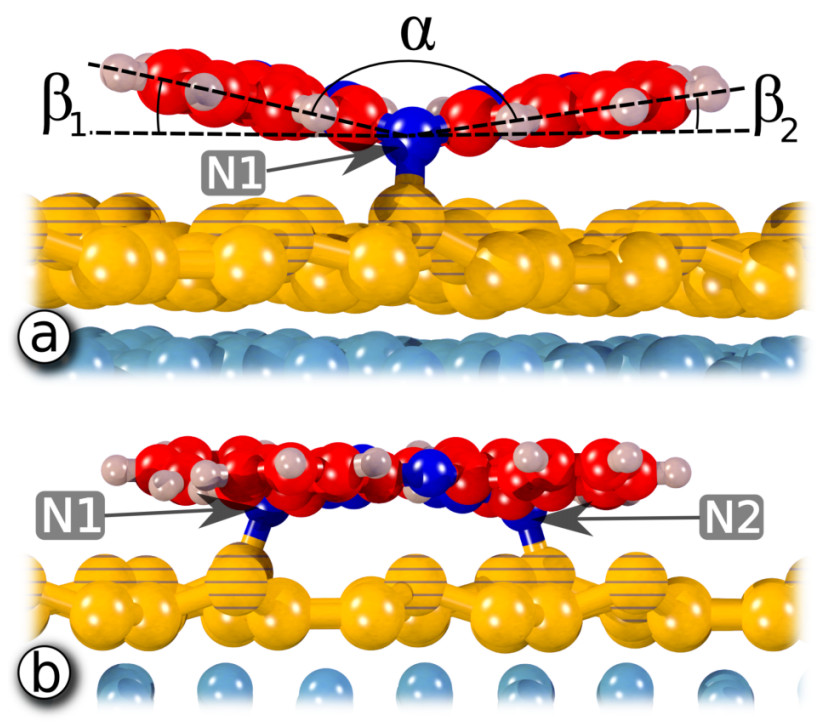

FIG. 2. Ball-and-stick model of the relaxed atomic structure of the $\mathrm{H}_{2} \mathrm{PC}$ molecule adsorbed on the $(3 \times 3)$ silicene $/(4 \times 4) \mathrm{Ag}(111)$ phase. (a) Side-view of Figure 1(c), showing the butterfly configuration of the $\mathrm{H}_{2} \mathrm{PC}$ molecule. (b) Side-view rotated by $90^{\circ}$ with respect to (a) and presenting the N1 and N2 atoms. decreases as shown in Table I. Finally, the $\mathrm{Si}-\mathrm{N}$ distances are rather short, ranging from $1.87 \AA$ to $1.94 \AA$.

Concerning the buckling of the silicene layer and the first $\mathrm{Ag}$ layer (the height difference between the top and bottom $\mathrm{Si}$ or $\mathrm{Ag}$ atoms), the value in the case of the silicene/Ag system within the DFT-D2 calculations is equal to $0.76 \AA$ and $0.33 \AA$, in agreement with DFT results obtained in previous works. ${ }^{32,38,68}$ How does the buckling vary in the presence of $\mathrm{H}_{2} \mathrm{Pc}$ on the silicene/Ag substrate? We note that the height difference between the $\mathrm{Si} 1$ and $\mathrm{Si} 2$ atoms and the bottom $\mathrm{Si}$ atoms $\left(\Delta_{\text {Sia }}\right.$ in Table I) lies between 0.99 and $1.06 \AA$, that is, higher than on pristine silicene. This result is in line with the possible formation of $\mathrm{Si}-\mathrm{N}$ covalent bonds. In contrast, the height of the four top Si atoms $\left(\Delta_{\mathrm{Sib}}\right.$ referred to the bottom $\mathrm{Si}$ atoms) located under the molecule phenyl rings decreases from $0.76 \AA$ to $0.52 \AA$, while in the other regions, the buckling changes very little $\left(\Delta_{\text {Sic }}=0.70-0.72 \AA\right)$. Now, as to the $\mathrm{Ag}$ buckling, it appears that the $\mathrm{Ag}$ atoms located below top $\mathrm{Si}$ atoms that have been pushed down upon molecule adsorption also shift downwards (see $\Delta_{\mathrm{Agb}}$ in Table I) with respect to their position in pristine silicene, while the other top $\mathrm{Ag}$ atom positions are only weakly modified. Finally, the DFTD2 calculations show that, for the different adsorption sites, the behavior of the molecule is similar: the molecule takes a butterfly conformation and the molecule-silicene distance is compatible with the formation of two covalent bonds.

In fact, the geometry of the molecule is similar to that of the benzene, the 1,3 cyclohexadiene, the 1,5 cyclooctadiene, or the biphenyl molecules in butterfly position on the $\mathrm{Si}(001)$ surface. ${ }^{15,18-25,69-72}$ In the case of the $\mathrm{H}_{2} \mathrm{Pc}$ molecule adsorption on the $\mathrm{SiC}$ and $\mathrm{SiB}$ surfaces, the structural study has shown that the $\mathrm{H}_{2} \mathrm{Pc}$ molecule stays nearly planar. ${ }^{26,27,30}$ Clearly, it is not the case for the adsorption of $\mathrm{H}_{2} \mathrm{Pc}$ on silicene/Ag. Nevertheless, the N1-N2 and $\mathrm{Si}-\mathrm{N}$ distances for these last systems $\left(7.16 \AA\right.$ and $1.88 \AA$ for $\mathrm{H}_{2} \mathrm{Pc} / \mathrm{SiC}$ and $6.50 \AA$ and 
TABLE I. Structural parameters calculated before (within parentheses) and after molecule adsorption by means of DFT-D2 method for the $\mathrm{H}_{2} \mathrm{Pc} / \mathrm{silicene} / \mathrm{Ag}$ system. The molecules angles denoted $\alpha, \beta_{1}$, and $\beta_{2}$ are displayed in Fig. 2. The atomic distances referred to as Si1 $\cdots \mathrm{Si} 2, \mathrm{Si} 1-\mathrm{N} 1, \mathrm{Si} 2-\mathrm{N} 2$, and N1 $\cdots \mathrm{N} 2$ are given in $\AA$. $\Delta_{S i a}, \Delta_{S i b}$, and $\Delta_{S i c}$ correspond to the height difference between the down Si atoms and the top Si atoms bonded to the molecule, the other top Si atoms located to the $\mathrm{H} 2 \mathrm{pc}$ molecule, and the remaining top Si atoms, respectively. $\Delta_{A g a}, \Delta_{A g b}$, and $\Delta_{A g c}$ indicate the Ag buckling of the Ag atoms located below the Sia, Sib, and Sic atoms.

\begin{tabular}{|c|c|c|c|c|c|c|c|c|c|c|c|c|c|}
\hline & $\alpha$ & $\beta_{1}$ & $\beta_{2}$ & $\mathrm{~N} 1 \cdots \mathrm{N} 2$ & $\mathrm{Si} 1 \cdots \mathrm{Si} 2$ & Si1-N1 & $\mathrm{Si} 2-\mathrm{N} 2$ & $\Delta_{S i a}$ & $\Delta_{S i b}$ & $\Delta_{S i c}$ & $\Delta_{A g a}$ & $\Delta_{A g b}$ & $\Delta_{A g c}$ \\
\hline Model 1 & $154(180)$ & $13(0)$ & $13(0)$ & $7.00(6.71)$ & $8.68(9.13)$ & 1.94 & 1.94 & 1.06 & 0.52 & $0.71(0.76)$ & 0.36 & 0.15 & $0.28(0.33)$ \\
\hline Model 2 & $150(180)$ & $16(0)$ & $14(0)$ & $6.73(6.71)$ & $7.93(8.14)$ & 1.89 & 1.87 & 0.99 & 0.52 & $0.70(0.76)$ & 0.23 & 0.14 & $0.25(0.33)$ \\
\hline Model 3 & $152(180)$ & $13(0)$ & $15(0)$ & $6.75(6.71)$ & $7.76(7.90)$ & 1.88 & 1.88 & 0.99 & 0.50 & $0.72(0.76)$ & 0.25 & 0.13 & $0.28(0.33)$ \\
\hline
\end{tabular}

$1.84 \AA$ for $\mathrm{H}_{2} \mathrm{Pc} / \mathrm{SiB}$ ) are very close to those obtained on the $\mathrm{H}_{2} \mathrm{Pc} /$ silicene/Ag one.

\section{B. Energetical study}

The adsorption energies for the three models, calculated following Equation (1), are $-2.50,-2.84$, and $-2.80 \mathrm{eV}$, respectively. These adsorption energies are very close to each other, and we conclude that the molecule can be stabilized on the silicene/ $\mathrm{Ag}$ substrate. Moreover, the calculations show that the adsorption energies for the rotated molecule by $90^{\circ}$ for the three sites do not change by more than $0.03 \mathrm{eV}$. So, the orientation of the molecule does not play a crucial role. Finally, in the following, we focus our study on site 2 presented in Fig. 1(c) that corresponds to the energetically most stable site.

The adsorption energy for site 2 is $-2.84 \mathrm{eV}$. For comparison, the adsorption energy of the $\mathrm{H}_{2} \mathrm{Pc}$ molecule on different surfaces, namely, the passivated $\mathrm{SiB}, \mathrm{SiB}$ with two boron defects, and $\mathrm{SiC}$, are, respectively, $-1.35 \mathrm{eV},-2.73 \mathrm{eV}$, and $-3.07 \mathrm{eV}^{26,27,30}$ In the case of the passivated SiB surface, the presence of boron atoms underneath the $\mathrm{Si}$ adatoms allows depopulation of the surface dangling bonds and one boron defect corresponds to the absence of a boron atom below one $\mathrm{Si}$ adatom. For all systems, the calculations have been carried on within the DFT-D2 method and with the same relaxation parameters (i.e., cut-off energy, force convergence, and k-point number), as already used in previous papers. ${ }^{26,27,30}$ It allows to define a stability scale of the $\mathrm{H}_{2} \mathrm{Pc}$ molecule on different Siterminated surfaces. The adsorption of the $\mathrm{H}_{2} \mathrm{Pc}$ molecule on the silicene/ $\mathrm{Ag}$ system is more stable than on the passivated $\mathrm{SiB}$ surface, and on the $\mathrm{SiB}$ surface with two boron defects, but less stable than on the SiC surface.

In order to characterize more precisely the energetic behavior of the $\mathrm{H}_{2} \mathrm{Pc}$ on $\mathrm{Si} / \mathrm{Ag}$ substrate, we now calculate the interaction energy following Equation (2). We find an interaction energy of $-4.27 \mathrm{eV}$ within $\mathrm{D} 2$ correction and $-1.00 \mathrm{eV}$ using only the PBE calculation. We note that the part of the van der Waals is important due to the size of the molecule and corresponds to $76 \%$ of the interaction energy. This large value of van der Waals energy is in agreement with other calculations. ${ }^{73,74}$ In order to understand why the adsorption and interaction energies are different, we calculate the deformation energy of the molecule and the substrate related to molecular adsorption. Indeed, before adsorption, the molecule is planar in gas phase and, after adsorption, the molecule takes a less stable butterfly conformation. The energy difference between both conformations is $+1.13 \mathrm{eV}$. On the other hand, the energy related to the geometric distortion of the substrate, due to the presence of the $\mathrm{H}_{2} \mathrm{Pc}$ molecule, is $+0.30 \mathrm{eV}$. This deformation of the silicene is related to the structural modification of the $\mathrm{Si}$ layer after molecular adsorption. Adding the interaction energy $(-4.27 \mathrm{eV})$, the deformation energies of the molecule $(+1.13 \mathrm{eV})$ and the substrate $(+0.30 \mathrm{eV})$, we find the value of $-2.84 \mathrm{eV}$ that corresponds to the adsorption energy. We conclude that the deformation of the molecule and the substrate is energetically very important for the $\mathrm{H}_{2} \mathrm{Pc} /$ silicene/ $\mathrm{Ag}$ system.

\section{Electronic structure}

The electronic study allows to obtain further information concerning the reaction mechanism of the $\mathrm{H}_{2} \mathrm{Pc}$ molecule adsorption on silicene. The charge density map reported in Fig. 3 shows clearly the presence of a high charge density between the molecule and the silicene, leading to the formation of two $\mathrm{Si}-\mathrm{N}$ bonds. The calculated ELF, presented in Fig. 4, is taken in the plane perpendicular to the silicene surface containing $\mathrm{N} 1$ and $\mathrm{N} 2$. The presence of an orange-red domain located around the Si2-N2 bond indicates strong electron localization, and thus the presence of a covalent bond. Note that the ELF at the Si1-N1 bond is similar to that of the Si2-N2 one. In order to highlight the electron redistribution driven by molecule adsorption, Fig. 5 presents a electron density difference map built as following: at first, the electron density has been calculated for the molecule/silicene/Ag system, the silicene/Ag system, and the molecule, without further atomic relaxation. Then, we extracted the electron density difference between the whole system on one side, and, on the other side, the bare silicene/Ag and the molecule. The side view of the electron density difference in Fig. 5(a) emphasizes, with the presence of a electron gain between $\mathrm{S} 1$ and $\mathrm{N} 1$ and between Si2 and N2 (light green plots), the formation of two chemical bonds. The top view of the electron density difference in

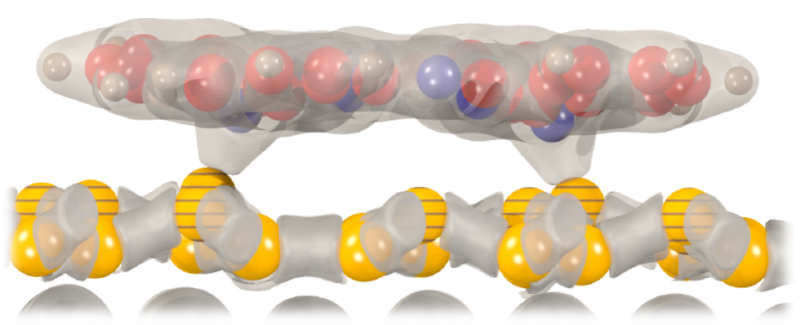

FIG. 3. Side-view of the charge density map (in translucent grey) at 0.50 $e^{-} / \AA^{3}$. C, N, H, top Si, down $\mathrm{Si}$, and Ag atoms are represented by red, dark blue, lightpink, striped yellow, yellow, and blue balls, respectively. 


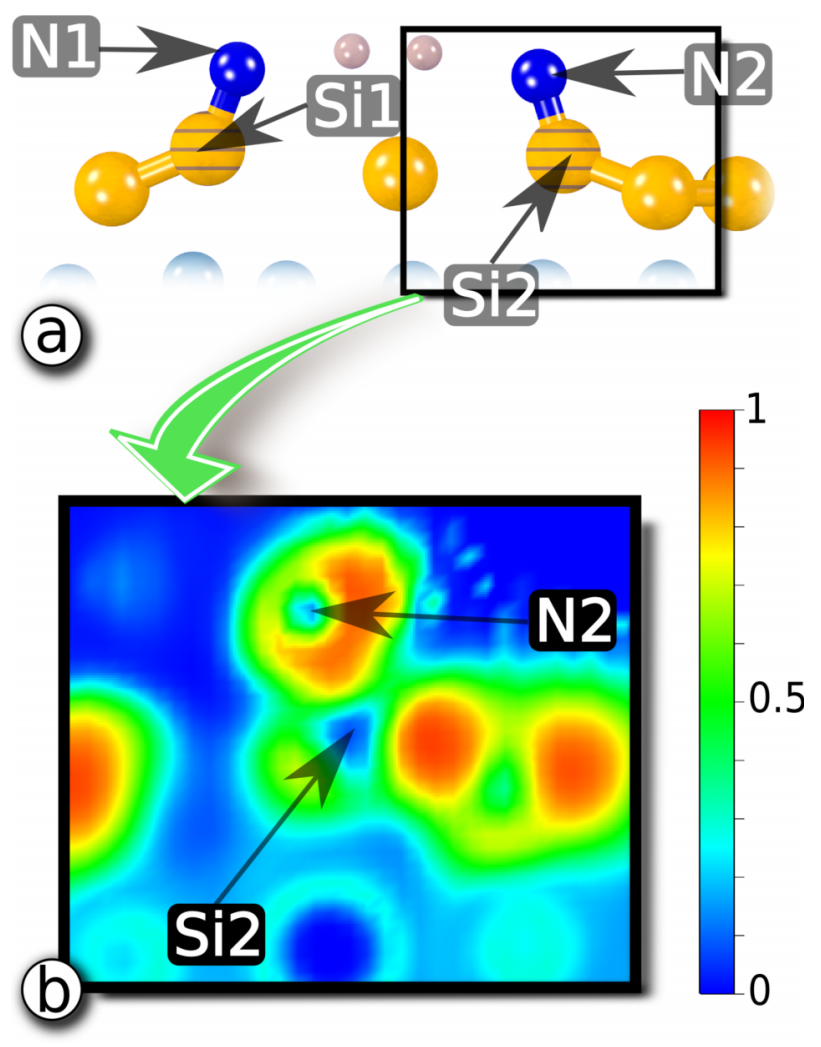

FIG. 4. (a) Side-view of the plane perpendicular to the surface and containing $\mathrm{N} 1$ and $\mathrm{N} 2$. N, top Si, down $\mathrm{Si}$, and $\mathrm{Ag}$ atoms are represented by dark blue, striped yellow, yellow, and blue balls, respectively. (b) Electron localisation function map taken in the area indicated in (a). Note that the ELF at the Si1-N1 bond (not presented here) is similar.

Fig. 5(b) shows a charge variation around the central macrocycle, which is alternatively positive and negative. The electronic redistribution in the central macrocycle of the $\mathrm{H}_{2} \mathrm{Pc}$ molecule, along with the formation of two $\mathrm{Si}-\mathrm{N}$ covalent bonds between the molecule and the silicene, is in agreement with a reaction of cycloaddition. Due to the distance between the two top $\mathrm{Si}$ atoms, the reaction can be assimilated to a $[10+2]$ pseudocycloaddition, as already observed on the $\mathrm{SiC}$ and $\mathrm{SiB}$ surfaces. ${ }^{26,27,30}$ However, a question still remains open: contrary to what happens on the $\mathrm{SiC}$ or $\mathrm{SiB}$ surfaces, why is the adsorbed molecule on silicene not planar? To answer this question, the Bader charges are also calculated in the $\mathrm{H}_{2} \mathrm{Pc} / \mathrm{Si} / \mathrm{Ag}$ system.

As reported in Table II, there is a charge transfer from the silicene to the $\mathrm{Ag}$ substrate and the $\mathrm{H}_{2} \mathrm{Pc}$ molecule. Moreover, the adsorption of the $\mathrm{H}_{2} \mathrm{Pc}$ molecule modifies only slightly the value of the charge gained by the Ag layers. Now, let us compare these results with those obtained on other substrates. The charge at the molecule is close to that obtained for the $\mathrm{H}_{2} \mathrm{Pc}$ on the $\mathrm{SiC}\left(-2.19|e|\right.$, where $\left.|e|=1.6 \times 10^{-19} \mathrm{C}\right)$ and two defects $\mathrm{SiB}(-2.27|e|)$. On passivated $\mathrm{SiB}$, the charge transferred to the molecule is only of $-0.09|e|$ due to the absence of chemical reaction between the $\mathrm{H}_{2} \mathrm{Pc}$ molecule and this substrate. In other words, the butterfly conformation of the $\mathrm{H}_{2} \mathrm{Pc}$ molecule cannot be explained by the charge transfer. We have to seek another cause of the molecule deformation on silicene.

Now, the distance between two nearest $\mathrm{Si}$ adatoms is equal to $9.29 \AA$ for the $\mathrm{SiC}$ surface and $6.70 \AA$ for the $\mathrm{SiB}$.

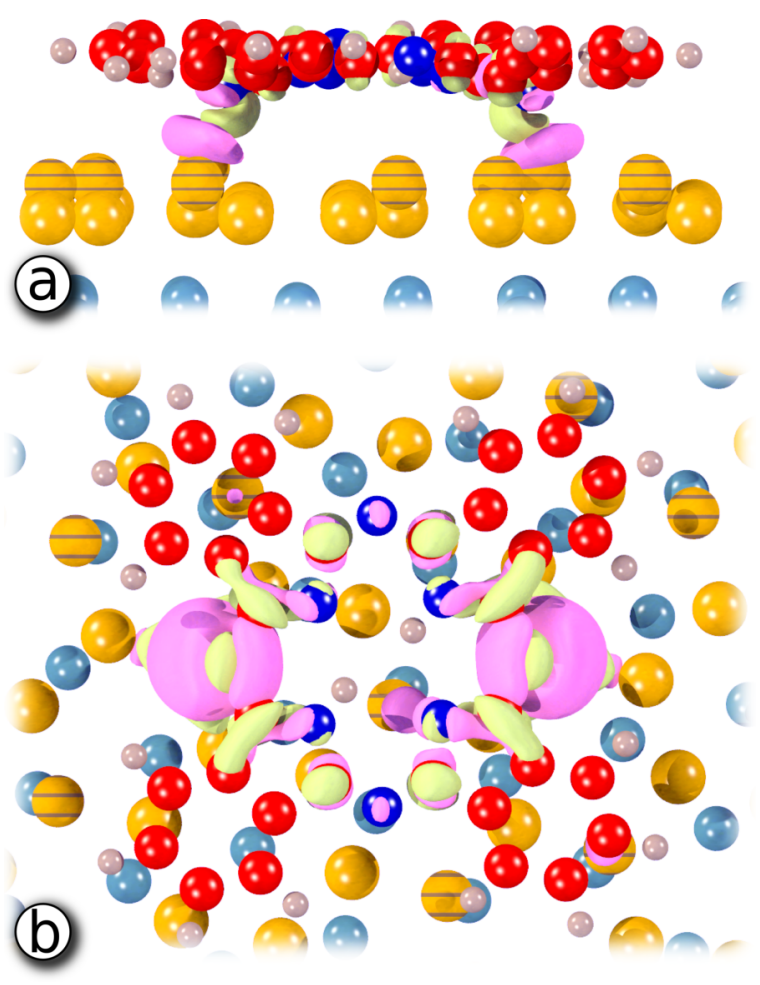

FIG. 5. Electron density difference maps displayed at (a) $0.04 e^{-} / \AA^{3}$ and (b) $0.015 e^{-} / \AA^{3}$ induced by $\mathrm{H}_{2} \mathrm{PC}$ deposition on the silicene $/ \mathrm{Ag}(111)$ surface. Light green and pink plots correspond to an augmentation and a diminution of the local electron density, respectively. C, N, H, top Si, down $\mathrm{Si}$, and $\mathrm{Ag}$ atoms are represented by red, dark blue, light pink, striped yellow, yellow, and blue balls, respectively. (a) Side-view and (b) top view, for clarity, only the first Ag plane is presented.

These distances are large enough to avoid the presence of $\mathrm{Si}$ adatoms under the $\mathrm{H}_{2} \mathrm{Pc}$ molecule. This is not the case for the silicene layer. In fact, the presence of top Si atoms under the phenyl cycles of the $\mathrm{H}_{2} \mathrm{Pc}$ molecule could explain the deformation of the molecule. Indeed, the vertical position of the top Si atoms located below the molecule is lower (see $\Delta_{\mathrm{Sib}}$ in Table I) than that of the other ones. This last observation could be related to the presence of repulsive interactions between both sides of the molecule and these top Si atoms. Finally, associated to the presence of top $\mathrm{Si}$ atoms under the molecule, in view of the electronic reorganization in the molecule and the silicene layer, we cannot exclude repulsive electrostatic or polarization effects between the molecule and the silicene layer.

\section{D. $\mathrm{H}_{2} \mathrm{Pc}$ adsorption on freestanding silicene}

In order to evaluate the possible influence of the $\mathrm{Ag}$ substrate, we now calculate the adsorption of the $\mathrm{H}_{2} \mathrm{Pc}$ molecule on

TABLE II. Calculated Bader charge in different parts of the $\mathrm{H} 2 \mathrm{Pc} / \mathrm{Si} / \mathrm{Ag}$ system in elementary charge $|e|$.

\begin{tabular}{lccc}
\hline \hline Bader charge & $\mathrm{H}_{2} \mathrm{Pc} /$ silicene/Ag & Silicene/Ag & $\mathrm{H}_{2} \mathrm{Pc} /$ freestanding silicene \\
\hline $\mathrm{H}_{2} \mathrm{Pc}$ & $-2.03|e|$ & $\ldots$ & $-2.06|e|$ \\
Silicene & $+5.27|e|$ & $+3.10|e|$ & $+2.06|e|$ \\
$\mathrm{Ag}$ & $-3.24|e|$ & $-3.10|e|$ & $\ldots$ \\
\hline \hline
\end{tabular}



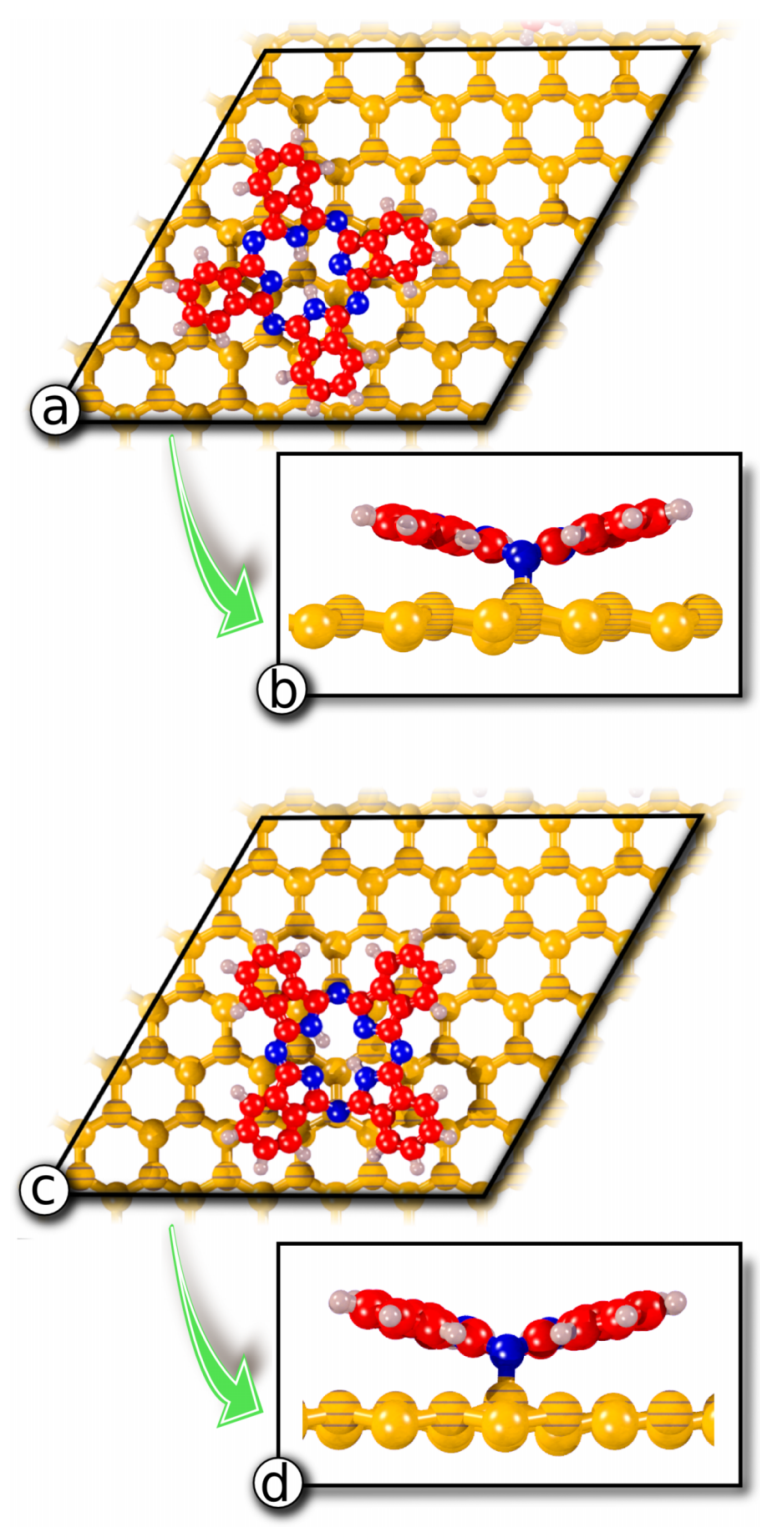

FIG. 6. (a) and (c) Top-view and (b) and (d) side view of the $\mathrm{H}_{2} \mathrm{Pc}$ molecule on the freestanding silicene layer for two different adsorption sites.

the freestanding silicene layer. Two different sites of molecular adsorption have been taken into account. The top and side views of the two models are presented in Fig. 6. We note that, in both cases, the molecule on the silicene layer takes a butterfly conformation as for the $\mathrm{H}_{2} \mathrm{Pc}$ adsorption on the silicene/ $\mathrm{Ag}$ substrate. The adsorption energies are $-1.45 \mathrm{eV}$ for the 1 st site (Figs. 6(a) and 6(b)) and $-1.41 \mathrm{eV}$ for the 2 nd one (Figs. 6(c) and $6(\mathrm{~d})$ ). For the two sites, the adsorption energies are close to each other. The molecule deformation energy for the most stable site (site 1) is $+1.49 \mathrm{eV}$, and the opening angle $\alpha$ is $146^{\circ}$. We note that this angle is smaller than that obtained for the adsorption on the silicene/Ag substrate $\left(150^{\circ}\right)$. The molecule is thus slightly more deformed on freestanding silicene, reflected in a higher deformation energy $(+1.49 \mathrm{eV}$ instead of $+1.13 \mathrm{eV}$ ). Finally, the charge transfer is equal to $2.06|e|$ from the silicene to the molecule (see Table II), very close to what is obtained on the silicene/ $\mathrm{Ag}$ surface. Thus, the final state of the $\mathrm{H}_{2} \mathrm{Pc}$ molecule is only marginally affected by the presence of the Ag substrate.
In all previous results, the D2 approximation has been taken into account. We also tested the $\mathrm{H}_{2} \mathrm{Pc}$ molecule adsorption on freestanding silicene within the PBE approximation only. After relaxation, the $\mathrm{H}_{2} \mathrm{Pc}$ molecule also takes a butterfly conformation. So, the latter does not depend explicitly on van der Waals interactions in the DFT calculations.

To summarize, we note that, without the $\mathrm{Ag}$ substrate, the $\mathrm{H}_{2} \mathrm{Pc}$ molecule still takes a butterfly conformation. We thus conclude that this molecular geometric deformation is the result of local interactions between the molecule and the silicene layer. Moreover, the butterfly conformation is not due to van der Waals interactions, and the electron density difference maps (Fig. 5) show that the sides of the molecule do not interact chemically with the silicene, since no covalent bonds are present between the phenyl rings and the Si atoms. Finally, we would like to stress that the most important difference between the $\mathrm{SiB}$ and $\mathrm{SiC}$ surfaces, where the adsorbed molecule is planar, and the silicene, where the molecule presents a butterfly conformation, is the presence of top $\mathrm{Si}$ atoms under the molecule. Thus, we suggest that electrostatic or polarization repulsions are present between the molecule and the top $\mathrm{Si}$ atoms of the silicene layer, in agreement with the complex electronic reorganization emphasized by the charge density difference (Fig. 5), and the fact that the four top Si atoms below the phenyl cycles are pushed downwards upon molecule adsorption.

\section{CONCLUSION}

We have studied the adsorption of the large $\mathrm{H}_{2} \mathrm{Pc}$ molecule on the silicene/Ag substrate in the framework of DFT-D2 calculations. We show that a pseudo-cycloaddition takes place with the formation of two covalent bonds between two $\mathrm{N}$ atoms of the $\mathrm{H}_{2} \mathrm{Pc}$ and two top Si atoms of the silicene. The electronic reorganization within the central macrocycle of $\mathrm{H}_{2} \mathrm{Pc}$ supports this adsorption reaction. The presence of the $\mathrm{H}_{2} \mathrm{Pc}$ molecule modifies locally, in particular under the molecule, the buckling of the silicene and the first Ag layer which decreases with respect to the bare silicene/Ag system. Moreover, contrary to the planar conformation of the $\mathrm{H}_{2} \mathrm{Pc}$ molecule observed on $\mathrm{SiB}$ or $\mathrm{SiC}$ surfaces, the molecule adopts a butterfly conformation on the silicene/Ag substrate. It appears that on freestanding silicene, the molecule still takes a butterfly conformation. The PBE calculation without D2 approximation shows that the butterfly conformation is not due to van der Waals interactions. Now, on $\mathrm{SiC}$ and $\mathrm{SiB}$ surfaces, where the molecule is planar, there are no top Si atoms below the molecule. However, on silicene, four top $\mathrm{Si}$ atoms are present that do not form covalent bonds with the molecule, and whose vertical position is lower than that of the other top Si. Thus, in order to explain the butterfly conformation of the $\mathrm{H}_{2} \mathrm{Pc}$ molecule on silicene/ $\mathrm{Ag}$ substrate, we suggest the presence of electrostatic or polarization repulsions between the sides of the $\mathrm{H}_{2} \mathrm{Pc}$ and the top $\mathrm{Si}$ atoms located below the molecule.

Finally, this DFT study opens the door to a possible chemisorption of large molecules on the silicene/Ag system. Nevertheless, only experiment, as already performed on the $\mathrm{SiC}$ surface, ${ }^{26,27}$ will allow to answer directly the question of the adsorption of large molecules on the silicene/Ag substrate. 


\section{ACKNOWLEDGMENTS}

This work was performed using HCP resources from GENCI-IDRIS and the supercomputer facilities of the Mésocentre of Strasbourg.

${ }^{1}$ O. Diels and K. Alder, Justus Liebigs Ann. Chem. 460, 98 (1928).

${ }^{2}$ O. Diels and K. Alder, Justus Liebigs Ann. Chem. 470, 62 (1929).

${ }^{3}$ O. Diels and K. Alder, Ber. Dtsch. Chem. Ges. 62, 2081 (1929).

${ }^{4}$ O. Diels and K. Alder, Ber. Dtsch. Chem. Ges. 62, 2087 (1929).

5J. T. Yates, J. Phys.: Condens. Matter 3, S143 (1991).

${ }^{6}$ M. J. Bozack, P. A. Taylor, W. J. Yates, and J. T. Yates, Surf. Sci. 177, L933 (1986).

${ }^{7}$ P. A. Taylor, R. M. Wallace, C. C. Cheng, W. H. Weinberg, M. J. Dresser, W. J. Choyke, and J. T. Yates, J. Am. Chem. Soc. 114, 6754 (1992).

${ }^{8}$ L. Clemen, R. M. Wallace, P. Taylor, M. J. Dresser, W. J. Choyke, W. H. Weinberg, and J. T. Yates, Surf. Sci. 268, 205 (1992).

${ }^{9}$ C. C. Cheng, R. M. Wallace, P. A. Taylor, W. J. Choyke, and J. T. Yates, J. Appl. Phys. 67, 3693 (1990).

${ }^{10}$ C. C. Cheng, W. J. Choyke, and J. T. Yates, Surf. Sci. 231, 289 (1990).

${ }^{11}$ M. J. Bozack, W. J. Choyke, L. Muehlhoff, and J. T. Yates, Surf. Sci. 176, 547 (1986).

${ }^{12}$ C. C. Cheng, S. Lucas, H. Gutleben, W. J. Choyke, and J. T. Yates, J. Am. Chem. Soc. 114, 1249 (1992).

${ }^{13}$ M. L. Cheng, M. L. Colaianni, and J. T. Yates, Surf. Sci. Lett. 274, L605 (1992).

${ }^{14}$ C. C. Cheng, Q. Gao, W. J. Choyke, and J. T. Yates, Phys. Rev. B 46, 12810 (1992).

${ }^{15}$ Q. Liu and R. Hoffmann, J. Am. Chem. Soc. 117, 4082 (1995).

${ }^{16}$ A. V. Teplyakov, M. J. Kong, and S. F. Bent, J. Am. Chem. Soc. 119, 11100 (1997).

${ }^{17}$ R. Konecny and D. Doren, J. Am. Chem. Soc. 119, 11098 (1997).

${ }^{18}$ J. S. Hovis, H. Liu, and R. J. Hamers, Surf. Sci. 402-404, 1 (1998).

${ }^{19}$ H. Liu and R. J. Hamers, J. Am. Chem. Soc. 119, 7593 (1997).

${ }^{20}$ J. S. Hovis, S. Lee, H. Liu, and R. J. Hamers, J. Vac. Sci. Technol., B 15, 1153 (1997).

${ }^{21}$ J. S. Hovis and R. J. Hamers, J. Phys. Chem. B 101, 9581 (1997).

${ }^{22}$ J. S. Hovis and R. J. Hamers, J. Phys. Chem. B 102, 687 (1998).

${ }^{23}$ R. J. Hamers, J. S. Hovis, S. Lee, H. Liu, and J. Shan, J. Phys. Chem. B 101, 1489 (1997).

${ }^{24}$ R. J. Hamers, S. K. Coulter, M. D. Ellison, J. S. Hovis, D. F. Padowitz, M. P. Schwartz, C. M. Greenlief, and J. N. Russel, Acc. Chem. Res. 33, 617 (2000).

${ }^{25}$ M. Mamatkulov, L. Stauffer, C. Minot, and P. Sonnet, Phys. Rev. B 73, 035321 (2006).

${ }^{26}$ G. Baffou, A. Mayne, G. Comtet, G. Dujardin, P. Sonnet, and L. Stauffer, Appl. Phys. Lett. 91, 073101 (2007).

${ }^{27}$ G. Baffou, A. Mayne, G. Comtet, G. Dujardin, L. Stauffer, and P. Sonnet, J. Am. Chem. Soc. 131, 3210 (2009).

${ }^{28}$ H. Yang, O. Boudrioua, A. Mayne, G. Comtet, G. Dujardin, Y. Kuk, P. Sonnet, L. Stauffer, S. Nagarajan, and A. Gourdon, Phys. Chem. Chem. Phys. 14, 1700 (2012).

${ }^{29}$ O. Boudrioua, H. Yang, P. Sonnet, L. Stauffer, A. Mayne, G. Comtet, G. Dujardin, Y. Kuk, S. Nagarajan, A. Gourdon, and E. Duverger, Phys. Rev. B 85, 35423 (2012).

${ }^{30}$ K. Boukari, E. Duverger, L. Stauffer, and P. Sonnet, Phys. Chem. Chem. Phys. 16, 12164 (2014).

${ }^{31}$ B. Lalmi, H. Oughaddou, H. Enriquez, A. Kara, S. Vizzini, B. Ealet, and B. Aufray, Appl. Phys. Lett. 97, 223109 (2010).

${ }^{32}$ C. Lin, R. Arafune, K. Kawahara, N. Tsukahara, E. Minamitani, Y. Kim, N. Takagi, and M. Kawai, Appl. Phys. Express 5, 45802 (2012).

${ }^{33}$ H. Jamgotchian, Y. Colignon, N. Hamzaoui, B. Ealet, J. Hoarau, B. Aufray, and J. Biberian, J. Phys.: Condens. Matter 24, 172001 (2012).

${ }^{34}$ P. Vogt, P. De Padova, C. Quaresima, J. Avila, E. Frantzeskakis, M. C. Asensio, A. Resta, B. Ealet, and G. Le Lay, Phys. Rev. Lett. 108, 155501 (2012).
${ }^{35}$ B. Feng, Z. Ding, S. Meng, Y. Yao, X. He, P. Cheng, L. Chen, and K. Wu, Nano Lett. 7, 3507 (2012).

${ }^{36}$ L. Meng, Y. Wand, L. Zhang, S. Du, R. Wu, L. Li, Y. Zhang, G. Li, H. Zhou, W. Hofer, and H. Gao, Nano Lett. 13, 685 (2013).

${ }^{37}$ P. Vogt, P. Capiod, M. Berthe, A. Resta, P. De Padova, T. Bruhn, G. Le Lay, and B. Grandidier, Appl. Phys. Lett. 104, 021602 (2014).

${ }^{38}$ R. Stephan, M. C. Hanf, and P. Sonnet, J. Phys.: Condens. Matter 27, 015002 (2015).

${ }^{39}$ A. Resta, T. Leoni, C. Barth, A. Ranguis, C. Becker, T. Bruhn, P. Vogt, and G. Le Lay, Sci. Rep. 3, 2399 (2013).

${ }^{40}$ C. L. Lin, R. Arafune, K. Kawahara, M. Kanno, N. Tsukahara, E. Minamitani, Y. Kim, M. Kawai, and N. Takagi, Phys. Rev. Lett. 110, 076801 (2013).

${ }^{41}$ D. Chiappe, C. Grazianetti, G. Tallarida, M. Fanciulli, and A. Molle, Adv. Mater. 24, 5088 (2012).

${ }^{42}$ R. Arafune, C.-L. Lin, K. Kawahara, N. Tsukahara, E. Minamitani, Y. Kim, N. Takagi, and M. Kawai, Surf. Sci. 608, 297 (2013).

${ }^{43}$ A. Kara, H. Enriquez, A. P. Seitsonen, L. C. Lew Yan Voon, S. Vizzini, B. Aufray, and H. Oughaddou, Surf. Sci. Rep. 67, 1 (2012).

${ }^{44}$ H. Oughaddou, H. Enriquez, M. R. Tchalala, H. Yildirim, A. J. Mayne, A. Bendounan, G. Dujardin, M. A. Ali, and A. Kara, Prog. Surf. Sci. 90, 46 (2015).

${ }^{45}$ L. Tao, E. Cinquanta, D. Chiappe, C. Grazianetti, M. Fanciulli, A. Molle, and D. Akinwande, Nat. Nanotechnol. 10, 227 (2015).

${ }^{46}$ J. Feng, Y. Liu, H. Wang, J. Zhao, Q. Cai, and X. Wang, Comput. Mater. Sci. 87, 218 (2014).

${ }^{47}$ G. Kresse and J. Hafner, Phys. Rev. B 47, 558 (1993).

${ }^{48}$ G. Kresse and J. Hafner, Phys. Rev. B 49, 14251 (1994).

${ }^{49}$ G. Kresse and J. Furthmüller, Comput. Mater. Sci. 6, 15 (1996).

${ }^{50} \mathrm{G}$. Kresse and J. Furthmüller, Phys. Rev. B 54, 11169 (1996).

${ }^{51}$ P. E. Blochl, Phys. Rev. B 50, 17953 (1994).

${ }^{52}$ G. Kresse and D. Joubert, Phys. Rev. B 59, 1758 (1999).

${ }^{53}$ J. P. Perdew, K. Burke, and M. Ernzerhof, Phys. Rev. Lett. 77, 3865 (1996).

${ }^{54}$ J. P. Perdew, K. Burke, and M. Ernzerhof, Phys. Rev. Lett. 78, 1396 (1997).

${ }^{55}$ S. Grimme, J. Comput. Chem. 27, 1787 (2006).

${ }^{56}$ S. Grimme, J. Antony, S. Ehrlich, and S. Krieg, J. Chem. Phys. 132, 154104 (2010).

${ }^{57}$ S. Grimme, S. Ehrlich, and L. Goerigk, J. Comput. Chem. 32, 1456 (2011).

${ }^{58}$ R. F. W. Bader, Chem. Rev. 91, 893 (1991).

${ }^{59}$ G. Henkelman, A. Arnaldsson, and H. Jònsson, Comput. Mater. Sci. 36, 354 (2006).

${ }^{60}$ D. Loffreda, Angew. Chem. 118, 6687 (2006).

${ }^{61}$ C. Liu, S. Zhang, P. Wang, S. Huang, and H. Tian, Int. J. Hydrogen Energy 38, 8367 (2013).

${ }^{62}$ M. J. Spencer, K. W. J. Wong, and I. Yarowsky, Mater. Chem. Phys. 119, 505 (2010).

${ }^{63}$ B. Silvi and A. Savin, Nature 371, 683 (1994).

${ }^{64}$ A. Savin, H. J. Flad, J. Flad, H. Preuss, and H. G. von Schnering, Angew. Chem., Int. Ed. Engl. 31, 185 (1992).

${ }^{65}$ W. Humphrey, A. Dalke, and K. Schulten, J. Mol. Graphics 14, 33 (1996).

${ }^{66}$ Theoretical and Computational Biophysics Group, Visual Molecular Dynamics software, Beckman Institute for Advanced Science and Technology, University of Illinois, http://www.ks.uiuc.edu/Research/vmd/.

${ }^{67}$ K. Momma and F. Izumi, J. Appl. Crystallogr. 44, 1272 (2011).

${ }^{68}$ P. Gori, O. Pulci, F. Ronci, S. Colonna, and F. Bechstedt, J. Appl. Phys. 114, 113710 (2013).

${ }^{69}$ P. Czekala, C. Panosetti, H. Lin, and W. Hofer, Surf. Sci. 621, 152 (2014).

${ }^{70}$ W. Hofer, A. Fisher, G. Lopinski, and R. Wolkow, Phys. Rev. B 63, 085314 (2001).

${ }^{71}$ W. Hofer, A. Fisher, G. Lopinski, and R. Wolkow, Surf. Sci. 482-485, 1181 (2001).

${ }^{72}$ J. Wieferink, P. Krueger, and J. Pollmann, Phys. Rev. B 78, 165315 (2008).

${ }^{73}$ H. Lange, J. Maulzsch, W. Meng, D. Mollenhauer, B. Paulus, N. Peica, S. Schlecht, and C. Thomsen, Langmuir 27, 7258 (2011).

${ }^{74}$ K. Boukari, P. Sonnet, and E. Duverger, ChemPhysChem 13, 3945 (2012). 